\title{
LA FORMACIÓN DEL PROFESORADO DE EDUCACIÓN FÍSICA: ALGUNAS CUESTIONES
}

\section{Autor}

\section{Resumen}

El artículo que a continuación se presenta tiene por finalidad constituir una base para el debate sobre posibles líneas de transformación y mejora de los procesos formativos del profesorado de Educación Física. La discusión se presenta articulada alrededor de tres ejes: la búsqueda de un marco filosófico que oriente y dote de sentido a los procesos formativos del profesorado; la adquisición de herramientas conceptuales que proporcionen al alumnado marcos de referencia amplios que le permitan contextualizar y problematizar la acción; y la toma de conciencia de que los procedimientos, nunca son neutros porque incorporan, de manera implícita y explícita, principios y valores que refuerzan un modelo de formación.

Palabras clave: Formación del profesorado, Teorias implícitas, Reconstrucción del conocimiento.

\section{Introducción}

Este trabajo constituye una parte de la tesis doctoral de la autora y en él se plantean, a la luz de las conclusiones del trabajo de investigación desarrollado, posibles líneas de avance para los procesos formativos del profesorado de Educación Física. A modo de contextualización diré que esta investigación se sitúa, dentro del ámbito formativo, en la formación del profesorado de EF. Y reúne en este espacio dos mundos que tienen que ver con lo que enseñamos en Educación Física y con la manera en que lo hacemos. Esta realidad constituye el objetivo principal de la investigación y se llevará a cabo a través del estudio de un programa formativo postgrado puesto en marcha y desarrollado en la CAV a través de diferentes convocatorias, para formar a maestros generalistas como maestros especialistas en Educación Física.

Mi participación en dicho programa me permitió entrar en contacto con una realidad social, para mi hasta ese momento desconocida a pesar de mi experiencia con el alumnado de formación inicial. Este contacto con colegas adultos supuso, no solo un gran avance personal y profesional, sino que hizo explícitos, a través de las importantes tensiones, conflictos surgidos y satisfacciones proporcionadas, una serie de interrogantes que se encontraban en mis reflexiones de una manera laten- te y que esta interacción impulsó, y que tienen que ver con las premisas y supuestos sobre los que se sustenta el currículum formativo, los significados e implicaciones de la orientación del programa formativo, el tipo de discurso sobre la Educación Física que la formación sanciona y los problemas y las dificultades con las que los docentes se encuentran en la práctica docente en el aula.

En la búsqueda de respuesta a estos interrogantes es donde encuentra su sentido y motivación la investigación, en un contexto profesional dominado por los estudios positivistas que obvian las perspectivas sociales y que generan entendimientos de la disciplina centrados básicamente en el rendimiento, donde el cuerpo es visto como un objeto a controlar y superar dentro de un ámbito biomecánico y fisiológico relacionado con una serie de competencias físicas orientadas hacia la productividad (Kirk, 1990; Devís, 1993, 1995; Ruso,1993; Sparkes, 1993; Fraile, 1995; Barbero, 1996; Fernández Balboa, 1997; Pascual, 1997); y la formación del profesorado, en coherencia con el entendimiento de la disciplina, como el desarrollo de técnicas y conductas eficaces que se suponen propias de esta profesión y en su eficaz aplicación (Tinning,1992; Evans y Penney,1993). 
Este entendimiento contrasta con nuestra creencia de que la EF. debe contribuir al conocimiento y comprensión de nosotros mismos como seres físicos y sociales al desarrollo de los aspectos fundamentales de la identidad individual y de la realidad subjetiva, a la calidad de vida, y a la participación crítica en las actividades de movimiento (Kirk, 1990 Tinning, 1993; Shilling, 1993) y de que en la formación del profesorado el fortalecimiento personal y social es éticamente previo a cuestiones epistemológicas, o al dominio de habilidades técnicas; que la formación requiere un compromiso con la transformación social para lo cual es necesario examinar en qué medida y bajo qué formas sirve para reproducir principios, valores y privilegios (Kirk, 1990: Dewar, 1993 Fernández Balboa, 1993; Tinning, 1993; Shilling, 1993).

Desde esta última perspectiva se articula esta investigación, la cuál, tomando como objeto de estudio el programa formativo postgrado de especialización en Educación Física desarrollado en la C.A.V., inicia su recorrido para buscar respuestas a los siguientes interrogantes:

- ¿Cuáles son las premisas y supuestos sobre los que se sustenta el currículum formativo desarrollado?

- ¿Qué orientaciones ha desarrollado el programa formativo: significados e implicaciones?

- ¿Qué temas han sido percibidos por los participantes en la formación como conflictivos a lo largo del proceso formativo?

- ¿Cuáles han sido las carencias, demandas y aciertos del proceso formativo expresadas por los participantes y las alternativas que proponen para su superación?

- ¿Cuál ha sido el impacto y las implicaciones del currículo formativo en la realidad de la práctica educativa del aula con relación a los discursos y propósitos educativos de la Educación Física?

- ¿Qué problemas expresan los participantes en la investigación con relación a la práctica docente en el aula?

- ¿Qué podemos proponer para futuros procesos formativos?

El último aspecto mencionado es el que se desarrolla en el presente artículo y, en esta dirección quiero resaltar que no es mi intención ofrecer, una vez detectados los fallos y bondades del programa desarrollado, una réplica de éste para aumentar su eficacia, sino elaborar una serie de argumentos de carácter básicamente normativos que proyecten y esbocen aspectos relevantes que deberían contemplar los procesos formativos del profesorado de Educación Física.

\section{Referentes teóricos $y$ consideraciones metodológicas}

La lente o el referente que guía la investigación la ha proporcionado el proyecto intelectual de la Teoría Crítica, en particular la creada por J. Habermas y los pedagogos que se han basado en su obra, Giroux (1987);
Carr y Kemmis (1988); Kemmis (1988); Grundy (1991) y Carr (1996). Estos autores proponen recuperar de la filosofía antigua aquellos elementos del pensamiento social que se ocupaban de los valores, juicios e intereses de la humanidad, para integrarlos en un marco de pensamiento que pudiera proporcionar a la ciencia social un nuevo planteamiento. Determinar cuándo las afirmaciones teóricas incluyen regularidades invariables de la acción social como tal y cuándo expresan relaciones de dependencia ideológicamente distorsionadas, que pueden ser transformadas, se convierte en su foco principal de atención.

En el momento actual la Teoría Crítica de la educación se enfrenta con el reto de proporcionar la base teórica necesaria para plantear enfoques alternativos a la organización escolar, al currículum, a la pedagogía en el aula y a las relaciones sociales; falta también por redefinir el papel de los actores (profesorado-alumnado), constituidos por múltiples subjetividades organizadas, asentadas sobre diferentes y complejas contradicciones.

Carr y Kemmis (1988), dos de las figuras más representativas en el ámbito de la enseñanza y la formación del profesorado a la luz de la Teoría Crítica, identifican una serie de condiciones que cualquier enfoque de teoría educativa debe tener, y que constituyen el referente teórico, a saber:

Debe cuestionar las nociones positivistas de racionalidad, objetividad y verdad para rechazar que el conocimiento tenga un papel exclusivamente instrumental en la resolución de los problemas del ámbito educativo porque supone reducir cualquier cuestión a un problema técnico.

En segundo lugar, apuntan la importancia de comprender los significados que las prácticas educativas tienen para aquellos que las llevan a cabo, esto supone admitir las categorías interpretativas de los docentes.

- Una tercera condición surge de la anterior y se relaciona con las limitaciones que presenta el modelo interpretativo si no se acompaña de los medios y las orientaciones apropiadas para distinguir aquellas autointerpretaciones que están ideológicamente distorsionadas de las que no lo están y que vienen determinadas por la estructura social sobre la que los docentes no ejercen una influencia directa.

- Una cuarta condición de la teoría educativa, tiene que ver con identificar aquellos aspectos que, derivados de los mencionados condicionantes, impiden la persecución de fines racionales.

- La quinta condición es la necesidad de reconocer que la teoría educativa es práctica, en el sentido de que la educación va a quedar determinada por la manera en que se relacione con la práctica, siendo la labor de la teoría la de informar y guiar las prácticas de los educadores, enfrentándose ambos aspectos de una manera dialéctica con el propósito de orientarse hacia la transformación.

En relación a la perspectiva o enfoque que ha seguido esta investigación y puesto que no es su propósito el descubrimiento de regularidades ni leyes universales, se ha considerado la opción cualitativa, a través del estudio de caso (el caso lo constituye el desarrollo de un 


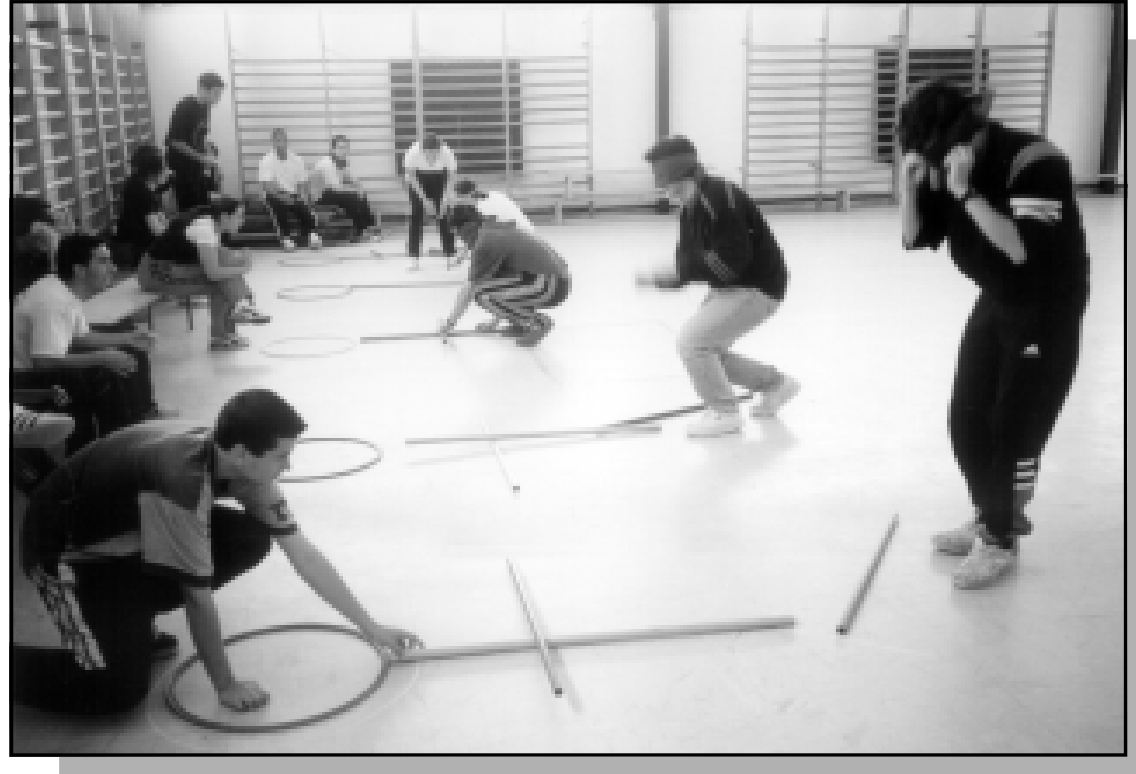

programa formativo postgrado llevado a cabo por un grupo de profesores en ejercicio en la enseñanza obligatoria) como la más adecuada para captar e interpretar las estructuras del mundo de la intersubjetividad de los participantes y reconstruir el significado del objeto de estudio desde la propia perspectiva del profesorado participante.

Desde la perspectiva que proporcionan los fines, el objeto, el enfoque de la investigación y el referente teórico, emprendemos la recogida de información. Asumir como referente teórico la Teoría Crítica ha obligado a incorporar a la investigación información sobre el contexto histórico, social, cultural y económico de la realidad a estudiar para percibir los influjos que recibe; a incorporar el concepto "ideología" para examinar el conjunto de opiniones que dominan en el campo; y la noción de praxis entendida como acción reflexión orientada a la transformación de la realidad; constituyendo las fuentes de información: los marcos teóricos y conceptuales del objeto de estudio, la documentación oficial, las entrevistas en profundidad y los diarios de clase.

La información proporcionada por los marcos teóricos ha permitido examinar y reflexionar sobre el contexto disciplinar, profesional y curricular de la formación del profesorado de Educación Física para tomar conciencia, a modo de contextualización, del universo de significados que dan sentido a las prácticas formativas y que permiten situarnos y comprender estas prácticas para vislumbrar la historia que se ha ido construyendo alrededor de las mismas. Estos marcos teóricos también han proporcionado claves de lo que supone abordar la formación del profesorado desde una perspectiva crítica ya que ésta se constituye en la referencia sustantiva a través de la que se enfoca y adquiere significado el objeto de estudio del presente trabajo.

La extensa documentación oficial de las diferentes convocatorias ha sido otra fuente importante de información y se encuentra constituida por los boletines of iciales, los programas de las diferentes asignaturas del programa formativo, los materiales curriculares, memorias etc. y han proporcionado importante información sobre el punto de vista institucional.
La información recogida a través de las entrevistas y los diarios de clase (de dos cursos académicos consecutivos) la ha proporcionado un grupo de nueve docentes participantes en la formación en las diferentes convocatorias.

Paralelamente a la recogida de información comienza el proceso de análisis ya que han sido fases interconectadas y condicionadas mutuamente. En relación con los criterios generales que han guiado el análisis interpretativo se señala que son criterios sociohermenéuticos que toman como referencia el marco conceptual que proporciona la Teoría Crítica para comprender y reconstruir el texto en su contexto social, en los planteamientos que contienen y los intereses que contemplan (Alonso,1998).

Desde estos planteamientos se ha desarrollado un proceso de categorización básicamente inductivo que ha permitido el establecimiento de un sistema categorial sobre el que se articula el informe interpretativo desarrollado. En relación con la dinámica analítica, ésta ha comenzado con una aproximación a la documentación oficial, la cual nos ha permitido obtener un sentido general de los principales temas que en ella se encuentran y establecer las primeras dimensiones o núcleos conceptuales que responden a los temas recurrentes en esta documentación. Con la intención de profundizar en estas dimensiones se aborda una nueva recogida de información que se obtiene a través de diarios de clase y entrevistas realizadas a nueve docentes que participan en la investigación.

El análisis de esta información proporciona por un lado, mas información sobre las dimensiones existentes y por otro, aporta nuevas dimensiones hasta ahora no contempladas. Esta dinámica inicial proporciona un primer sistema categorial y constituye una primera espiral caracterizada por un análisis global, para retomar, con todas las dimensiones obtenidas, una lectura más precisa que desemboca en una revisión y redefinición de las dimensiones o metacategorías, para proceder posteriormente a su identificación, definición y la adscripción de las categorías en cada dimensión.

Este proceso es contrastado con colegas expertos surgiendo el sistema categorial definitivo alrededor del cual se ha articulado el informe interpretativo. Este sistema categorial queda constituido por cinco núcleos o metacategorías que recogen cinco direcciones diferentes de estudio y que tienen que ver con todos aquellos aspectos que rodean el programa formativo; con el propio programa, con la forma de experimentar el programa desde la perspectiva de los participantes, con los propósitos educativos de la disciplina y con las dificultades que suscita la acción educativa en el aula; y diecinueve categorías que se articulan en cada núcleo y que agrupan, dentro de cada núcleo, los aspectos relevantes y recurrentes para el objeto de estudio. 
La elaboración del informe interpretativo ha supuesto, a partir del examen y reflexión de los datos obtenidos en cada categoría, resituar el objeto de estudio en su contexto, exponer, justificar y desvelar el enfoque formativo desarrollado, su impacto en la docencia y las dificultades asociadas; así como plantear direcciones de avance en futuros procesos formativos, aspecto que expone y da cuenta este artículo.

\section{Implicaciones formativas}

Con el contexto anteriormente mencionado como marco de referencia y a la luz del informe interpretativo y en relación con el punto que desarrolla este artículo (qué podemos proponer para futuros procesos formativos) emergen tres focos que deben ser contemplados en las intervenciones formativas orientadas a formar docentes en Educación Física: el marco filosófico de la formación de profesorado en Educación Física, las herramientas conceptuales, y las cuestiones de procedimiento.

\section{El marco filosófico}

Un elemento clave que se vislumbra a lo largo de la investigación es la necesidad de construir, en comunidad, un marco filosófico que sirva de guía y referencia última a la Educación Física y, en coherencia, al proceso formativo. Esto significa un entramado de acuerdos compartidos, logrados por las personas que se encuentran involucradas en el proceso, que se articulan con otras creencias y concepciones que pueden justificarse desde puntos de vista morales. Esta tarea cobra especial relevancia al ser una materia donde una tradición socializadora poderosa ha construido una imagen cultural en la que su potencial educativo, lejos de símisma, se encuentra en sus posibilidades para contribuir a través de ella a fines fundamentalmente relacionados con la socialización.

Construir un marco filosófico supone reflexionar sobre la Educación, la Educación Física y la Formación, sobre el conocimiento acumulado en los diferentes campos de investigación y las hipótesis que en ellos se encuentran y sus significados ocultos para plantear pre-

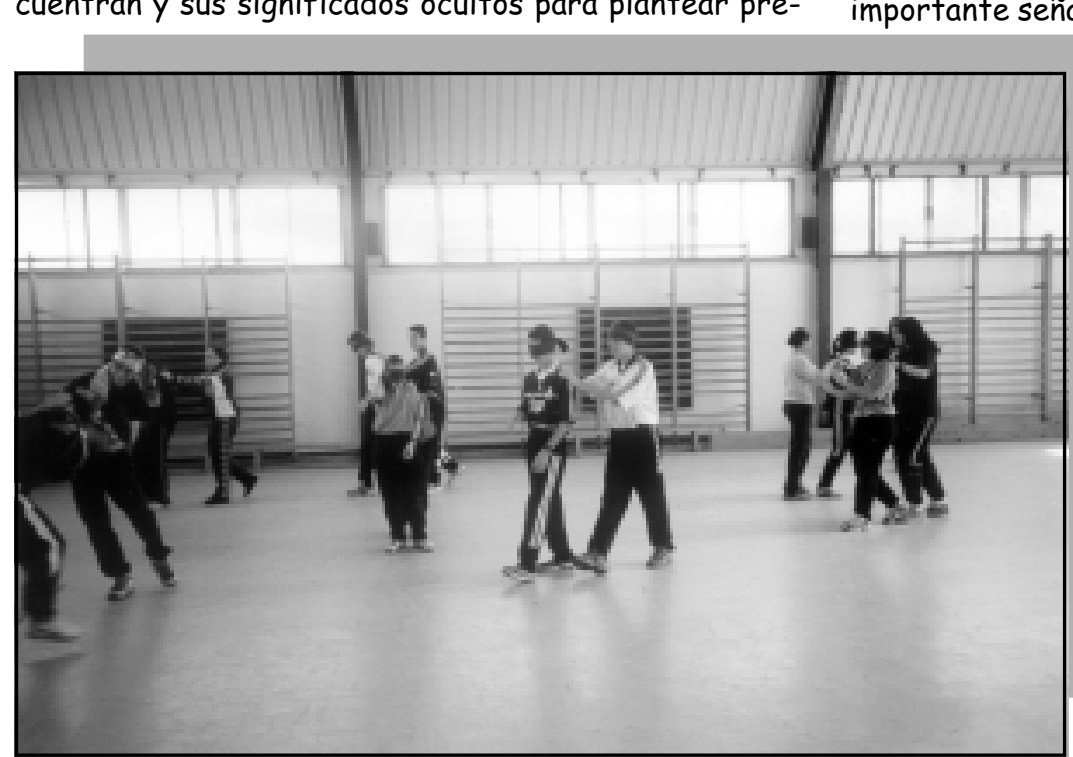

guntas fundamentales, aquellas que tienen que ver con los valores o concepciones de lo deseable, con principios y elecciones morales para, desde esta perspectiva, dar un mayor sentido y referencia al proceso educativo profesional y sentar las bases para organizar los puntos de vista y las creencias respecto a la Educación, la Educación Física y la Formación de su profesorado (Liston y Zeichner, 1993; Carr, 1996).

Esta construcción deberá superar muchos puntos de vista de la postmodernidad sobre la corporeidad que se basan en la cultura efectiva tecnológica occidental y en valores de individualismo y etnocentrismo que orientan a la adquisición de cuerpos dóciles, hábiles, capaces y eficientes. Para contribuir a la educación nuestra área debe proporcionar marcos alternativos que se enfoquen hacia el desarrollo de la identidad individual y la realidad subjetiva; así como hacia formas complejas de comunicación social y expresión, para contribuir a ampliar el conocimiento y entendimiento de los alumnos sobre ellos mismos como seres físicos, sociales y emocionales, mejorando su autoestima y autorrealización, e incorporando capacidades de análisis que posibiliten su participación crítica en una cultura del movimiento que, en el momento actual, constituye un referente destacado en la vida de las personas y frente a la que es necesario mostrar una actitud crítica debido al impacto cultural que tienen en nuestro mundo (Kirk, 1990; Arnold, 1991; Silvennoinen, 1993).

Este marco deberá centrarse en cuestionar y reconstruir los presupuestos sociales que afectan a los cuerpos y a las formas en que éstos son valorados, para aprender a habitar nuestros cuerpos con confianza profundizando en el conocimiento del mismo percibido desde el interior, en lugar de trabajar sobre ellos desde el exterior, desde su superficie para construir una apariencia que proyecte una imagen satisfactoria de nosotros (Dewar, 1993; Shilling, 1993; Bertherat y Bernstein, 1999).

En esta construcción jugarán un papel esencial las dimensiones "acerca del movimiento" $y$ "en el movimiento" señaladas por Arnold (1991), porque ofrecerán comprensión acerca de la actividad motriz que se desarrolla, las intenciones de ésta y su significado dentro del contexto social y cultural, impulsando una participación que estimule el conocimiento práctico. En este planteamiento es importante señalar que la actividad física no conduce al desarrollo cognitivo, sino que exige el conocimiento, entendimiento y conciencia conceptual como aspectos necesarios, aunque no suficientes, para una participación y resolución de la actividad motriz planteada (Kirk, 1990).

Parte de la responsabilidad de la elaboración de este marco filosófico recae sobre el propio proceso formativo y las personas que en él participan. El punto de partida requiere que la formación del profesorado de Educación Física supere la idea de un cuerpo biológico anatómico a modo de máquina formada por piezas separadas, orientado hacia el producto, rendimiento y control, para centrar su atención en la dimensión comunicativa, en la 
aceptación y respeto por las diferencias entre las personas, considerando las propias contingencias como positivas. Esta consideración del cuerpo, intenta explotar las posibilidades de que sea visto como sujeto, en vez de como objeto ya que nuestro cuerpo somos nosotros mismos (Bertherat y Bernstein, 1999).

Desde este marco filosófico la formación del profesorado se orientará a desarrollar tanto los aspectos de la enseñanza como moral, como los de oficio (Tom, 1994), haciendo explicito el propósito de impulsar y enriquecer la capacidad crítica del alumnado acerca de lo que está haciendo e indagar acerca de su propio pensamiento para desde esta comprensión, abordar la cultura profesional y social con la que toma contacto y se socializa.

Este planteamiento induce a pensar en la importancia del segundo foco planteado, las herramientas conceptuales. Al examinar, a través de diversos marcos de referencia la información que se recibe, el alumnado puede empezar a tratar el conocimiento como algo problemáticoy, de esta forma, como objeto de indagación.

\section{Herramientas conceptuales}

Los programas de formación de profesorado de Educación Física tienen que proporcionar al alumnado las herramientas teóricas y conceptuales necesarias para concebir el conocimiento como algo problemático, condicionado históricamente y creado socialmente, ofreciéndole la oportunidad de crear y reconstruir sus propios significados (Giroux, 1997).

En este sentido se indica que la formación del profesorado de Educación Física necesita superar la exposición sistemática de conocimientos seleccionados de la historia y la cultura y presentados como no problemáticos y exentos de valor; ocultando que, generalmente, presentan de una manera universalizada, normas, valores y puntos de vista dominantes que representan perspectivas interpretativas y normativas sobre la realidad social.

La mayoría de los programas de formación del profesorado de Educación Física se encuentran inmersos en el lenguaje y supuestos de las ciencias exactas; esta situación nos proporciona, para interpretar el mundo, unas herramientas conceptuales de aquello que se puede medir y cuantificar, a la vez que se constituye en indicativo de lo que es realmente valioso. Es necesario superar esta visión unidireccional para incluir la perspectiva que nos proporcionan las ciencias sociales y situar la disciplina dentro del contexto histórico, político, cultural económico y social, ya que es evidente que el devenir de la enseñanza de la disciplina no se desarrolla al margen del contexto, sino que éste se configura en un indicador que determina y articula los significados.

Los programas tienen que capacitar, a través de las perspectivas que abren la historia, la sociología, la antropología, la economía, la filosofía y la epistemología de la Educación Física, una mayor comprensión y contextualización de la disciplina para aprender a comprender desde otras lentes la relación de la Educación Física con la producción de relaciones de poder dominantes. La raza, el género, la clase, la edad, la sexualidad y la incapacidad son expresadas en y a través del cuerpo,y el profesorado participa de una manera central en la producción y generación de conocimiento y experiencias corporales (Giroux, 1990; Popkewitz, 1990; Dewar, 1993; Liston y Zeichner, 1993; Apple, 1966; Pascual, 1997).

Adoptar esta perspectiva requiere revisar los supuestos que han determinado el enfoque tecnocrático de la Formación del Profesorado de Educación Física caracterizado por la primacía de una serie de materias relacionadas con las ciencias naturales, y otras, vinculadas a nuestro ámbito específico y conformadas por los diferentes deportes, donde el cuerpo y el movimiento son contemplados como una destreza, una cuestión técnica que comienza con el aprendizaje de una serie de habilidades básicas, que evolucionarán hasta la adquisición de otras habilidades específicas conformadas por códigos de movimientos considerados más complicados que se han de aprender para, en el futuro, poderlos enseñar.

En relación con estas herramientas conceptuales señalar la importancia de la Historia en la formación del profesorado, ya que permitirá comprender cómo se forman las historias culturales a través del estudio de las practicas educativas específicas como construcciones históricas ligadas a acontecimientos económicos, sociales y políticos (Popkewitz, 1990). Otros aprendizajes relevantes tienen que ver con los Estudios Culturales, estudios que nos permitirán analizar los diferentes discursos que se producen en la cultura dominante. En nuestro ámbito, existe un complejo fenómeno cultural, social y económico, en relación al cuerpo y el movimiento como objeto de consumo, ocio, bienestar, salud..., que, ayudado por los medios de comunicación, trasciende a todos los ámbitos y colonializa nuestro pensamiento, razón por la cual es necesario que el profesorado aprenda a pensar críticamente sobre ello y a actuar en consonancia (Popkewitz, 1990).

Otro elemento que permitirá analizar los Estudios Culturales tiene que ver con la dignificación de culturas subordinadas. En nuestro caso, la cultura femenina es una cultura que se presenta en clara subordinación a la masculina. El capital cultural de la materia, históricamente producido, ha sido construido específicamente por hombres, esta posición dominante se deja sentir, de acuerdo con Conell (1997), porque una vez producido el conocimiento es necesario seleccionarlo para elaborar un currículum y esta selección "no lo hace una comisión de ángeles epistemológicos en el cielo" sino que lo realiza la cultura dominante, seleccionando y sancionando lo que constituirá las destrezas básicas y las áreas nucleares para encarnar en el aula esta cultura que prima determinados modelos de disciplina, rendimiento, eficacia y poder.

En definitiva las herramientas conceptuales ayudarán a desvelar cómo la cultura dominante representa determinadas lecturas y principios pedagógicos sobre la Educación Física y el Deporte como fenómenos culturales y sociales de una gran trascendencia, los cuales ejercen una gran influencia en todas las esferas de la vida, que los educadores tienen que comprender para ser capaces de configurar su propio pensamiento crítico.

\section{Las cuestiones de procedimiento}

Profundamente relacionados con todo lo que hasta el momento se ha planteado, e igualmente relevantes, se encuentran las cuestiones de procedimiento curricular. 
Un primer punto de inflexión se encuentra en el hecho de que cualquier procedimiento no es neutral porque no sólo está cargado de valor, sino que representa formas ideológicas determinadas de entender el mundo, y por tanto, supone un importante aprendizaje explícito e implícito que debe integrarse de manera consciente en el desarrollo del proceso formativo.

La forma en que el profesorado y el alumnado desempeñan las diferentes tareas debe atender a una serie de cuestiones, implícitas en el marco filosófico, que apuntan hacia:

- Favorecer procesos de reflexión que articulen la teoría y la práctica, rechazando la perspectiva instrumental acerca de que la teoría es algo que producen agentes ajenos a la escuela, y ayudando al alumnado a crear sus teorías prácticas a través de la consideración de la enseñanza como una forma de investigacióny al profesorado como investigador de su propia práctica; ambos capaces de producir conocimientos que puedan contribuir a su desarrollo profesional (Liston y Zeichner, 1993). Estos procesos requerirán examinar la práctica profesional de la Educación Física para descubrir las contradicciones y consecuencias que tienen que ver con las tensiones entre lo afectivo y lo cognitivo, las artes y las ciencias, lo cualitativo y lo cuantitativo, las exigencias de la excelencia y las diferencias entre escolarización y educación.

- Incorporar las diferentes individualidades a la situación pedagógica. Es necesario desarrollar enfoques pedagógicos que capaciten para entender el valor y la experiencia que cada persona, en relación a su vivencia aporta al aula en relación a la actividad física, para lo cual es importante desarrollar actividades encaminadas a profundizar en sus biografías con objeto de comprender cómo su propio capital cultural representa una construcción surgida de la dialéctica entre su experiencia personal y su historia. Este enfoque suministra las bases para entender cómo sus propios valores determinan las experiencias con las que trabajan. Motivar al alumnado para: explicitar, clarificar y articular sus concepciones en torno al cuerpo, al movimiento y la función de ambos en la educación y en la formación; analizar las influencias culturales presentes en esa construcción; tomar conciencia de las construcciones de los otros, para interrogarse y comprender cómo han sido creadas en su proceso de desarrollo personal $e$ individual.

- Establecer ambientes de aprendizaje cooperativos que estimulen la creación de espacios donde se elaboren propuestas, donde se negocien y discutan alternativas en el ejercicio de la democracia y autonomía que permitan tomar decisiones, en colaboración, sobre los contenidos, las actividades a desarrollar, la elaboración de materiales, la forma de la evaluación. Esta idea cuestiona el valor de la competición, tan vinculada a la Educación Física por el importante impacto que tiene en ella el ámbito deportivo, y que por definición, supone el logro individual.

- Establecer relaciones sociales que impulsen las relaciones humanas, por tanto emocionales, con las personas que forman parte del proceso formativo. Este planteamiento obliga a considerar la forma y el contenido de las relaciones sociales del aula entre los docentes y el alumnado requiriendo la apertura de

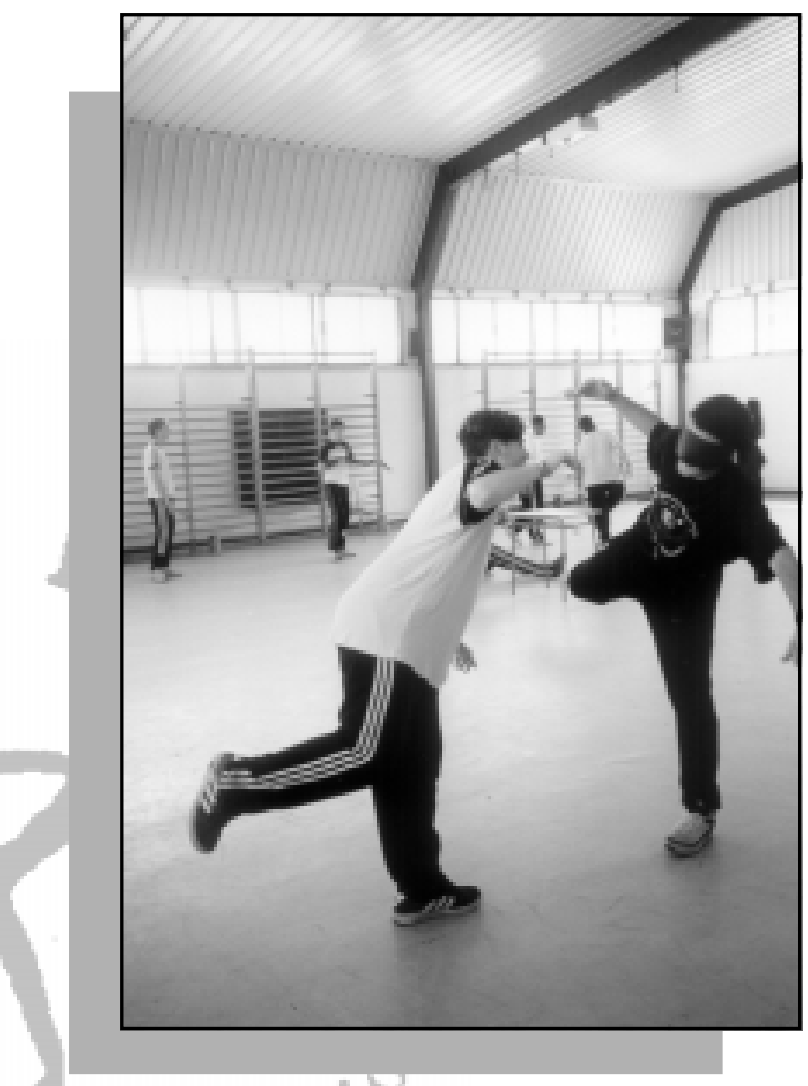

canales de comunicación que tengan en cuenta si existen las mismas posibilidades de hablar y de plantear cuestiones en la situación de aprendizaje para todos los participantes (Grundy, 1991), sin olvidar que también se establecen relaciones a través de la emoción, expresión corporal, contacto corporal..., aspectos que deberán ser contemplados.

- Desarrollar una cultura de la evaluación en coherencia con la acción educativa que se promueva, potenciando criterios y estrategias que tengan en cuenta todos los aspectos y colectivos que intervienen en el proceso.

\section{A modo de conclusión}

A modo de conclusión plantearé que las posibles propuestas formativas que se aborden, al margen de la estrategia desde la cual se encuentren diseñadas, deberán impulsar procesos a través de los cuales se adquiera una toma de conciencia sobre la intencionalidad de su acción, ya que cualquier programa formativo, aunque tenga la apariencia de una tarea desinteresada e impersonal, supone siempre un compromiso con una filosofía, y por tanto, con los valores educativos que esa filosofía lleva consigo. Por tanto, y a pesar de que las personas comprometidas con la formación pretendan abordar ésta sin articular ninguna creencia filosófica ni valor educativo, esto no significa que su trabajo no este impregnado de una filosofía y de los valores que ésta lleva implícitos. Partir de esta premisa es necesario para posibilitar una intervención más crítica en la práctica formativa y educativa, porque todo proceso formativo contiene, de forma más o menos explícita, una manera de entender la Educación Física y la formación del profesorado de Educación Física al servicio de un concepto de mundo y de hombre. 
En este sentido será necesario tener en cuenta que todo el conocimiento que se transmite al profesorado, tanto a través de la forma y de los contenidos, como a través de las relaciones sociales que se establecen entre el docente y el alumnado, no representan una descripción parcial de las cosas, sino que es el reflejo, al menos implícito, de cuestiones normativas respecto a la naturaleza de la educación, de la Educación Física y de la función de la Educación Física en la sociedad. Es por esto por lo que las cuestiones abordadas se constituyen en categorías necesarias para abordar un proceso formativo.

\section{Referencias Bibliográficas}

Alonso, L.E. (1998).La mirada cualitativa en sociología. Madrid: Fundamentos.

Appel, M. (1986). Ideología y currículum. Madrid: Akal. Morata.

Apple, M. (1996). Política cultural y educación. Madrid.

Arnold, P.J. (1991). Educación Física, movimiento y currículum. Madrid: Morata.

Barbero, J.I.: (1996). Cultura profesional y currículum (oculto) en Educación Física. Reflexiones sobre las (im)posibilidades del cambio. Revista de Educación, (311) 13-49.

Bertherat, TH y bernstein, C. (1999). El cuerpo tiene sus razones. Barcelona: Paidós.

Beyer, L. y zeichner, K. (1990). "La educación del profesorado en el contexto cultural: Más allá de la reproducción» en POPKEWITZ, Th. Formación del Profesorado. Madrid: Morata

Bourdieu, P. (1986). "Notas provisionales sobre la percepción social del cuerpo", en AA.VV.. Materiales de sociolo gía crítica. Madrid: La Piqueta.

Carr, W. y kemmis, S. (1988). Teoría crítica de la enseñanza .Barcelona. Martínez Roca.

Carr, W. (1990). Hacia una ciencia crítica de la educación. Barcelona: Laertes.

Carr, W. (1996). Una teoría para la educación. Madrid: Morata.

Connell, R.W. (1997). Escuelas y justicia social. Madrid: Morata.

Devís, J. (1993). Introducción crítica a la investigación positivista en la enseñanza de la Educación Física. En Inves tigación Alternativa en Educación Física, apuntes 227,7-30.

Devís, J. (1995). La investigación en la enseñanza de la Educación Física. EnRevista de Educación Física 58,35-38.

Dewar, A. (1993). El cuerpo marcado por el género en la Educación Física: una perspectiva feminista crítica. Investigación Alternativa en Educación Física. UNISPORT (227)111-126.

Eisner, E. (1998). El ojo ilustrado. Indagación cualitativa y mejora de la práctica educativa. Barcelona: Paidos.

Evans, J. y penney, D. (1993). La política de la pedagogía. En Investigación alternativa en Educación Física. UNISPORT Apuntes227, 127-142.

Fernandez Balboa, J.M. (1993). Aspectos crítico y cívico del rol de los/las profesionales de la Educación Física y el Deporte: Conexiones con la política, la economía y el medio ambiente. Apunts, (34) 74-82.

Fernandez Balboa, J.M (1997) La investigación en la Educación Física española. Un índice para el futuro. En Apunts 50,100-106.
Fraile Aranda, A. (1995). El maestro de educación física y su desarrollo profesional. Salamanca: Amarú.

García Ruso, H.(1993): La formación del profesorado de Educación Física: problemas y expectativas. Barcelona: Inde.

Giroux, H. (1987). La Formación del Profesorado y la ideología del control social. Revista de Educación, (284) 53-76.

Giroux, H. y MCLAREN, P. (1990). "La educación del profesorado como espacio contrapúblico", en POPKEWITZ, Th. (Ed) Formación del profesorado. Madrid: Morata.

Giroux, H. (1997). Intelectuales públicos y la crisis de la enseñanza superior. Revista Universitaria de Formación del Profesorado (29) 77-87.

Giroux, H. (1990). Los Profesores como intelectuales. Barcelona: Paidos.

Greene, M. (1987). Lo que exige lo conocido. Una orientación filosófica para la Formación del Profesorado. Revista de Educación (284) 125-131.

Grundy, S. (1991). Producto o Praxis del Curriculum. Madrid: Morata.

Kemmis, S. (1988). El currículum: más allá de la teoría de la reproducción. Madrid: Morata.

Kirk, D. (1990). Educación Física y Currículum. Valencia: Universidad de Valencia.

Liston, D. Y Zeichner, K. (1993). Formación del profesorado y condiciones sociales de la escolarización. Madrid: Morata.

Pascual, C. (1997). Análisis contextual en la Formación del Profesorado de Educación Física. Revista de Educación. (313) 161-178.

Pérez Gómez, A.I. (1993). La formación del docente como intelectual comprometido. Signos,( 8-9) 42-53.

Popkewitz, Th. S. (1990). Formación del profesorado. Madrid: Morata.

Popkewitz, Th. S. (1994). Sociología Política de las Reformas Educativas. Madrid: Morata.

Shilling, C. 1993). Cuerpo, escolarización y teoría social: el capital físico y la política de enseñanza de la educación física. Investigación Alternativa en Educación Física. UNISPORT (227) 93-110.

Silvennoinen, M. (1993). iTodos en fila!. La experiencia de la EF escolar y la imagen del papel del profesor. Investigación Alternativa en Educación Física. Málaga: Unisport.

Sparkes, A. (1993). Problemas éticos en la investigación de paradigmas alternativos, de la dinámica del compromiso a la política de la representación. En Investigación Alternativa en Educación Física. UNISPORT 227, 73-92

Tinning, R. (1992). La Educación Física: la escuela y sus profesores. Valencia: Universidad de Valencia.

Tinning, R. (1993). Reflexiones en torno a la investigación sobre la enseñanza reflexiva en la Formación del Profesorado. Investigación Alternativa en Educación Física. UNISPORT, (227) 159-182.

Tinning, R. (1996). Discursos que orientan el campo del movimiento humano y el problema de la Formación del Profesorado. Revista de Educación, (311) 123-134.

Tom, A.R. (1994). Conocimiento e interrogantes pedagógicos. Cuadernos de Pedagogía, (225) 79-83.

Vaquero Barba, A. (2002). Claves para la formación del profesorado de Educación Física desde una perspectiva crítica. Bilbao: UPV. 